\title{
¡Un, dos, tres por los niños escondidos en el pasado! Hacia una bioarqueología de la infancia en Colombia*
}

\author{
Playing Hide-and-Seek with the Children in the Past: Towards a \\ Bioarchaeology of Childhood in Colombia
}

\author{
Laura Coy Carrera* \\ Universidad Nacional de Colombia, Colombia \\ Claudia Rojas-Sepúlveda ${ }^{* * *}$ \\ Universidad Nacional de Colombia, Colombia
}

D0I: $10.22380 / 2539472 X .1051$

\begin{abstract}
RESUMEN
Los niños siempre han estado presentes en la historia de la humanidad y son un porcentaje importante de la población; sin embargo, han sido muy poco estudiados en la arqueología colombiana. Este artículo de revisión pretende re-conocer el potencial investigativo de la infancia y exponer algunas salidas metodológicas y teóricas a las dificultades con las que se encuentran los investigadores a la hora de estudiar a estos individuos a través del registro arqueológico $y$, particularmente, del bioarqueológico. Este enfo-que diferencial en la arqueología apenas comien-za a abrirse espacio en América Latina.
\end{abstract}

Palabras claves: infancia, bioarqueología, arqueología de la infancia, Latinoamérica.

\begin{abstract}
Children have always been present in the history of humanity and make up a significant percentage of the population, however, they have received little attention in Colombian archaeology. This review article aims to recognize the research potential of childhood and expose some methodological and theoretical alternatives to the difficulties that researchers face when studying these individuals in the archaeological records and, particularly, the bioarchaeological ones. This differential ap-proach in archaeology is recently emerging in Latin America.
\end{abstract}

Keywords: childhood, bioarchaeology, archaeology of childhood, Latin America.

El título hace alusión a la frase usualmente utilizada por los niños colombianos en el juego del escondite, con el propósito de hacer referencia a la búsqueda de los niños del pasado, ocultos bajo tierra o con un papel secundario, casi invisible, en la investigación arqueológica. Esta obra está bajo licencia internacional Creative Commons Reconocimiento-NoComercialSinObrasDerivadas 4.0

* $\quad$ Magíster en Antropología, línea Arqueología y Bioantropología, de la Universidad Nacional de Colombia. Pertenece al Grupo de Investigación en Antropología Biológica (GIAB) de la misma universidad. https://orcid.org/0000-0003-0747-1107 / ltcoyc@unal.edu.co

... Doctora en Antropología Biológica de la Aix-Marseille Université. Investigadora del Grupo de Investigación en Antropología Biológica (GIAB) de la Universidad Nacional de Colombia y profesora de la misma universidad. https://orcid.org/0000-0001-7471-1428 / cmrojass@unal. edu.co 


\section{¿De qué se trata el juego? Una breve introducción ${ }^{1}$}

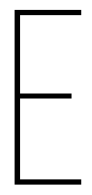

$\mathrm{n}$ todas las poblaciones humanas encontramos una gran cantidad de niños que han desempeñado papeles cruciales en diferentes momentos de la historia y que, llegado el momento, fueron los encargados de perpetuar las costumbres y saberes de sus grupos y ancestros (Hirschfeld 2002; Lillehammer 1989). Sin embargo, casi no los hallamos en los registros históricos, como si no hubiesen estado presentes; además, cuando revisamos las temáticas usuales trabajadas en la antropología y la arqueología, es notable su ausencia como sujetos de estudio hasta hace tan solo algunas décadas (Hirschfeld 2002; Montgomery 2009; Sacchi 2010).

Más allá de visibilizar la presencia de los niños en las sociedades del pasado, este artículo pretende reconocer y demostrar el potencial investigativo que tienen estos individuos en la bioarqueología colombiana y contribuir a un tema que ha ganado fuerza en otros lugares del mundo. En Colombia, la bioarqueología ha sido vista por largo tiempo como una disciplina poco útil, pues la antropología ha privilegiado el estudio de ámbitos considerados de mayor relevancia para las luchas de los pueblos vulnerables. Sin embargo, ha presentado un interesante avance después de la década de 1970. El regreso de investigadores formados en el extranjero permitió que se empezaran a realizar trabajos sobre restos óseos y dentales, particularmente del área muisca en el altiplano cundiboyacense, después en el Valle del Cauca y recientemente en la zona norte del país y el Alto Magdalena. Dichos estudios han aportado conocimiento sobre la forma de vida de los pueblos pre-hispánicos del actual territorio colombiano (Rodríguez 1996; Rodríguez-Flórez y Rodríguez-Flórez 2010; Rojas-Sepúlveda 2014). De manera mucho más reciente se han abordado poblaciones históricas, principalmente de la ciudad de Bogotá (Rivera 2014; Rojas-Sepúlveda y Rodríguez 2017). De todos modos, la investigación sobre los niños ${ }^{2}$ en la arqueología ha sido precaria, pues usualmente han estado incluidos como elementos que acompañan las dinámicas de los adultos y no como eje central de las investigaciones (Rivera 2004).

\footnotetext{
1 Es necesario agradecer a los profesores Javier Rivera y Lourdes Márquez por permitir un diálogo que fortaleció las ideas que presenta el artículo. También agradecemos al equipo editorial de la revista y a los tres evaluadores anónimos por sus excelentes observaciones. 


\section{¿Quiénes juegan? Repensando los conceptos de edad e infancia}

Es necesario aclarar que de modo general existen dos perspectivas diferentes para conceptualizar la edad: la edad biológica y la edad social del individuo. Asociados al primer tipo se encuentran conceptos propios de la bioantropología, la psicología y la pediatría, basados en las diferentes fases de desarrollo biológico y psicosocial de los individuos; en dichas fases, previas a la pubertad y adultez, ocurre el desarrollo físico a partir de las condiciones y necesidades físicas y ambientales del entorno (Fernández 2010; Lewis 2007; Pinhasi y Mays 2008). En segundo lugar, tenemos las definiciones socioculturales acordes con los diferentes contextos sociales e históricos en que se encuentran los individuos, a partir de las cuales se entiende la infancia como el periodo de aprendizaje de las normas culturales y en el que los niños incorporan reglas para vivir en sociedad (Fernández 2010; Halcrow y Tayles 2011, 347). La combinación de estas miradas nos permite entender con mayor claridad el panorama de la infancia en el pasado (Halcrow y Tayles 2011, 350; Perry 2006).

Dado que las normas culturales y las condiciones físicas en las que viven los individuos no son universales, los niños transitan por procesos particulares que se conjugan con las interpretaciones que los adultos hagan de ellos. Es por esa razón que la infancia es entendida como una categoría social que va más allá de los caracteres biológicos inamovibles y las definiciones universales; de hecho, se tiende a definir a partir de cada contexto específico (Lewis 2007). La infancia y el rol de los niños en la sociedad son un fenómeno cambiante, una construcción social, subjetiva y particular que debe ser estudiada a partir de las condiciones físicas, ambientales y sociales en las que se desarrolla la vida de estos sujetos (Sacchi 2010). Cada sociedad establece cómo se organiza y se entiende el periodo de la infancia, y genera un sinfín de modelos y categorizaciones. Las respuestas a preguntas como qué es ser niño o niña o cuándo comienza y termina la infancia varían según orientaciones culturales, y otras relativas a la clase social, al género del individuo, al momento histórico, entre otros factores. De este modo, la infancia se convierte en un fenómeno social atravesado por múltiples variables, que además puede ser asignado, manipulado, negado o revocado. 


\section{¿Han estado siempre escondidos? Algunas visiones sobre la infancia}

Para entender la relevancia de la infancia en los debates arqueológicos actuales es necesario un breve recorrido por el desarrollo de esta temática dentro de la disciplina. Especialmente porque, a pesar de que pocos trabajos se han enfocado en los niños, sí podemos rastrear su presencia desde los inicios de la antropología.

En el siglo XIX, la teoría de la evolución generó un interés por el desarrollo y transformación del individuo y de la especie, que llevó a pensar que estudiar el tránsito de un niño desde la infancia hasta la madurez podría reflejar el proceso de la especie humana desde el salvajismo hasta la civilización (Montgomery 2009). Autores como Edward Tylor veían la infancia como un fenómeno presocial y de naturaleza "salvaje", por lo que estudiar al niño permitiría entender la evolución de la humanidad contemporánea (Montgomery 2009; Pávez 2012). Bajo ópticas semejantes, los académicos del momento estudiaron la infancia y su "salvajismo", en oposición a la cultura del mundo civilizado europeo y norteamericano (Hirschfeld 2002). Según autores como Hirschfeld (2002) y Montgomery (2009), estas comparaciones entre el pensamiento de los niños y el pensamiento primitivo tuvieron un impacto negativo en el desarrollo posterior de la antropología de la infancia, pues ante el temor del resurgimiento de estas comparaciones, se terminó evadiendo el tema de la infancia como ámbito de teorización.

A finales del siglo XIX y comienzos del XX, la antropología física vio a estos individuos como objetos de estudio a los que aplicar las múltiples técnicas de medición que sirvieran para comparar niños de diferentes orígenes, con el propósito de argumentar a favor o en contra de los paradigmas evolucionistas y raciales del momento (Little y Sussman 2010; Montgomery 2009; Pachón 2009a). Sin embargo, la tendencia evidente en este tipo de investigaciones es que la infancia no se consideraba mucho más allá del espécimen para la toma de medidas antropométricas.

Unas décadas después, entre 1930 y 1970, bajo el enfoque de la llamada escuela de cultura y personalidad, se hicieron investigaciones como las de Margaret Mead, centradas en refutar el determinismo biológico universal y resaltar el papel de la cultura en el desarrollo de la personalidad adulta y el impacto de las experiencias de la infancia (Lancy 2012; Pachón 2009a). Aunque investigadores asociados a estas corrientes han recibido críticas por interesarse más en la transmisión de patrones culturales o por los sistemas de parentesco que por los propios niños o las ideas sobre la infancia, este enfoque es considerado uno de 
los principales referentes en el tema (Montgomery 2009), pues, además de cuestionar los roles de género universales y la supuesta similitud entre la mentalidad primitiva y el pensamiento de la infancia, casi por primera vez, la ubica como objeto central en la investigación antropológica (Harris 1979; Montgomery 2009).

La falta de reconocimiento que se le da a la producción cultural de los niños (Hirschfeld 2002) y la idea de que estos aún eran individuos incompletos o parcialmente socializados los convirtieron en sujetos poco útiles para quienes deseaban conocer la cultura adulta, vista como "completa” (Montgomery 2009, 6). Dichas ideas, combinadas con las dificultades metodológicas que podían presentarse a la hora de trabajar con niños - en comparación con los adultos-, como los problemas de comunicación o la posibilidad de que estos no comprendieran determinadas preguntas, o el hecho de que para los adultos el mundo de la infancia sea tan distante (en tanto más ligado a la imaginación o por considerarlos desconocedores de la cultura), contribuyeron a que el tema de la infancia siguiera relegado en la literatura antropológica.

En la década de 1960 notamos un cambio dentro de las ciencias sociales con Philippe Ariès (1987) y su obra El niño y la vida familiar en el Antiguo Régimen, en la cual estudia el surgimiento y desarrollo del concepto de infancia en la sociedad europea. Este trabajo, pionero en su campo, le dio a Ariès el título de "padre fundador de la historia de la infancia”, pues destacó que este concepto corresponde a una categoría social que varía espacio-temporalmente, que define el trato hacia los niños y cuya transformación —en este caso en Europa-puede ser estudiada a partir de las evidencias históricas.

No obstante, aun cuando los planteamientos de Ariès han sido criticados por hacer generalizaciones sobre la actitud de los adultos hacia los niños, sin explorar a profundidad la vida de estos individuos, y por centrarse únicamente en las clases altas europeas, la manera como conceptualiza la infancia y como utiliza diferentes fuentes históricas - entre estas la iconografía de la época-para entender los cambios dentro de un núcleo de organización social considerado familia y el modo de comprender la infancia abrieron las puertas a una temática de investigación que había sido poco explorada hasta el momento y que inició una serie de reflexiones sobre las diversas formas en que la niñez fue asumida en el pasado (Mays et al. 2017; Pachón 2009a; Pollock 1990).

Este interés por la infancia hace parte de un nuevo enfoque que se estaba gestando dentro de disciplinas como la historia, en la que las investigaciones dejaron de centrarse únicamente en los grandes acontecimientos y personajes del pasado, y extendieron su mirada hacia la vida privada y cotidiana de las personas del común, en donde los niños habían permanecido escondidos (DeMause 1982; Justel 2012). 
Dentro de este nuevo ámbito encontramos trabajos como el de Lloyd DeMause (1982), escrito con el propósito de entender la evolución de la infancia desde la antigua Grecia hasta mediados del siglo XX en Europa y Norteamérica, a partir de una postura psicoanalítica. Para este autor, el concepto de infancia fue asumido de formas diferentes, que pueden ser estudiadas basándose en reacciones psicológicas ${ }^{3}$ que determinan las diversas formas de relación entre adultos y niños en la historia. Según él, en esta relación existieron seis grandes momentos: el infanticidio (presente desde la antigua Grecia hasta el siglo IV), cuando la vida del niño era propiedad del padre, quien podía decidir sobre ella sin ningún inconveniente. El abandono (entre los siglos IV y XVIII), una práctica común de los padres, que no participaban directamente en la crianza de sus hijos sino que los abandonaban o enviaban fuera de la casa para aprender un oficio o educarse. La ambivalencia (entre los siglos XIV y XVII), caracterizada por el ingreso de los niños en la vida afectiva de los padres a pesar de ser considerados rebeldes a los que había que reprender y moldear. La intrusión (siglo XVIII), por su parte, surgió de un especial interés en el niño, quien ya no estaba tan lleno de las proyecciones ${ }^{4}$ que habían tenido los adultos, sino que, por el contrario, requería más proximidad de los padres. En este periodo también nació la pediatría que, junto con la mejora general de los cuidados por parte de los padres, redujo la mortalidad infantil. En el marco de la socialización, entre el siglo XIX y mediados del siglo XX, la crianza de un hijo pasó del intento de dominio de su voluntad a procurar formarlo y guiarlo, al tiempo que fue considerado mano de obra barata y como un bien a explotar. Por último, en la ayuda, que surgió a mediados del XX, se consideró que el niño sabía mejor que el padre lo que necesitaba en cada etapa de su vida y que requería involucrar la plena participación de ambos padres en la crianza, haciendo un esfuerzo por satisfacer sus necesidades peculiares y crecientes. Sin embargo, este autor no profundizó en las variaciones regionales o de clase que podrían afectar esta secuencia, y mucho menos exploró el trato hacia la niñez fuera de Europa Occidental y Norteamérica.

Como él, otros estudiosos analizan la historia de la infancia bajo el presupuesto de que existió una relación hostil y distante frente a los niños, en la cual

DeMause (1982) define tres tipos de reacciones al respecto: 1) la reacción proyectiva, en la que se usa al niño como vehículo para la proyección de los contenidos de su propio inconsciente; 2) la reacción de inversión, en la que el niño se usa como sustituto de una figura adulta importante en su propia infancia; y 3) la reacción de empatía, en la que se experimenta empatía respecto a las necesidades del niño y se actúa para satisfacerlas.

4 Desde una postura psicoanalítica, el autor plantea que hasta este periodo existió una relación proyectiva, de modo que el niño fue usado como vehículo para la proyección de los contenidos del inconsciente de sus padres (DeMause 1982). 
el abandono, el infanticidio y la muerte accidental a causa del descuido o malos cuidados por parte de los padres eran comunes (DeMause 1982; Dunn 1982; Sevilla 2012). Algunas investigaciones, por ejemplo, describieron de qué manera el infanticidio y el abandono fueron vistos como prácticas normales en Europa hasta después de finalizada la Edad Media, especialmente en lugares como la antigua Roma, en donde el padre de familia tenía poder decisorio sobre la vida y la muerte de sus hijos, por lo que era común dejar morir a los niños que no quería reconocer jurídicamente bajo su tutela (Sevilla 2012); entretanto, Dunn (1982)5, a través de análisis psicológicos de fuentes primarias y secundarias en inglés y en ruso, halla una serie de condiciones y tratos hostiles por parte de los adultos hacia la infancia rusa de los siglos XVIII y XIX; dentro de las costumbres identificadas por el autor, se encuentran la imposición de una rígida disciplina, castigos físicos y prácticas que desde la pediatría moderna podrían ser cuestionadas, por ejemplo el proceso de fajadura que restringía al máximo la movilidad de los bebés, o someter al niño al frío o calor extremos con el propósito de "endurecer" su carácter (Dunn 1982, 425). En contraste, Linda Pollock (1990) plantea que entre los años 1500 y 1900 también existieron relaciones de empatía y cuidado por parte de los padres. En su texto Los niños olvidados estudia los vínculos entre padres e hijos ingleses y norteamericanos en ese largo periodo a partir de 433 fuentes, entre diarios, memorias, autobiografías y cartas, que le permiten cuestionar la idea de que los padres no estaban interesados o preocupados por sus hijos, con lo que puso de manifiesto exactamente lo opuesto. Sin embargo, aun cuando la autora indica que recopiló registros de diferentes personas de diversas clases sociales y ocupaciones - desde la aristocracia inglesa hasta campesinos-, estos textos necesariamente se limitan a la población que sabía leer y escribir, lo que sin duda genera vacíos a la hora de entender los modelos de crianza y de relacionamiento entre los adultos y los niños en esas épocas y lugares.

Para el siglo XVII también se encuentran evidencias de un creciente interés por la educación y formación del niño. Por ejemplo, en 1658 se publicó por primera vez un libro ilustrado con fines didácticos para niños, el Orbus sensalium pictus, encaminado a la enseñanza del latín que surge como respuesta a las transformaciones que se estaban presentando en torno a la institución de la escuela (Aguirre 2001).

Fuera de Europa y Norteamérica, otras sociedades han conceptualizado de formas distintas la infancia, por ejemplo, en el léxico del antiguo Egipto

$5 \quad$ Nótese que el nombre del texto en inglés es: “The Enemy is the Baby': Childhood in Imperial Russia”, algo diferente a la traducción española: “'Ese enemigo es el niño': la infancia en la Rusia imperial". 
existieron cerca de treinta vocablos para referirse a un niño (Justel 2012). Además, a estos individuos se les han asignado diferentes papeles sociales, que incluso los han ubicado en posiciones de poder; bastaría solo mencionar el caso de $\mathrm{Pu}$ Yi, quien con menos de tres años fue nombrado emperador de China (Ríos 1998), o el caso del faraón egipcio Tutankamón, quien asumió el poder cuando tenía entre ocho y diez años (Grimal 1996).

Estos ejemplos nos permiten dimensionar un vasto repertorio de formas de conceptualizar y relacionarse con la infancia que varían cultural e históricamente (Montgomery 2009). A estas variaciones tendríamos que sumarles las particularidades de cada individuo o de cada grupo dentro de determinada sociedad, lo cual impide realizar generalizaciones aun dentro de un mismo periodo histórico. Además, las diferencias de género, clase social e incluso de edad pueden incidir en los modos en que los individuos viven aquello concebido como infancia.

Muchos de los estudios realizados hasta el momento centraron su atención en la relación entre padres e hijos, y no propiamente en los niños, quienes siguieron viéndose como un objeto pasivo dentro de las estructuras familiares y sociales de cada periodo (Justel 2012). Fue con los estudios de género y con el feminismo que se empezó a investigar sobre sujetos hasta el momento considerados "invisibles", como las mujeres y los niños (Mays et al. 2017). Surgieron entonces perspectivas como la planteada por Hardman (2001), en el marco de las cuales se reconoce que los niños son personas que deben estudiarse por derecho propio y que cuentan con sus propias formas de pensar y de ver el mundo, así como con sus propios conocimientos culturales. En este sentido, Lancy (2012) llama la atención sobre la necesidad de generar un mayor diálogo entre las investigaciones que han tomado a la infancia como su objeto de estudio con la intención de generar un cuerpo teórico e intelectual de la antropología de la infancia. Del mismo modo, autoras como Pachón (2009a) y Pollock (1990) reconocen el largo camino que aún falta por recorrer y la necesidad de generar más estudios interdisciplinarios sobre la infancia, para sortear las dificultades propias de trabajar con este segmento de la población. Sin embargo, en las últimas décadas los estudios sobre la niñez han reposado en colaboraciones mutuas de disciplinas como la antropología, la historia, la medicina, la demografía, la psicología, entre otras. 


\section{¿En nuestra historia y antropología también han estado escondidos los niños?}

Como se describió, gran parte de las discusiones se han centrado en estudiar grupos específicos de niños en Europa y Norteamérica, mientras que en América Latina sigue siendo un tema que requiere ser explorado con mayor profundidad. Encontramos pocas investigaciones sobre la historia de la infancia, pero se destaca una de gran relevancia: la compilación de trabajos realizada por Pablo Rodríguez y María Emma Mannarelli en el 2007, titulada Historia de la infancia en América Latina. En esta obra se presenta un panorama de la infancia desde tiempos prehispánicos hasta el siglo XX y se resalta cómo la infancia ha sido el sector poblacional menos visible en la historia latinoamericana, acostumbrada a los relatos nacionalistas, de héroes y gobernantes. El texto además muestra que la infancia en la región se encuentra determinada por factores como el contexto espacio-temporal, la filiación étnica y la clase social.

En Colombia, se destaca la compilación de Rodríguez y Mannarelli (2007), en la cual encontramos varios trabajos sobre la infancia que abarcan desde el periodo colonial hasta el siglo XX y versan, por ejemplo, sobre la participación de los niños en diferentes enfrentamientos bélicos en el país, la situación de los niños abandonados, el desarrollo de la educación infantil, la pediatría y la literatura infantil. Como afirma Pachón (2005), también tienen notoriedad los trabajos de Virginia Gutiérrez de Pineda (1978), quien dedicó su actividad investigativa a dos temáticas: la medicina popular y la familia colombiana. Si bien Gutiérrez de Pineda no trabajó específicamente el tema de la infancia, sus aportes al conocimiento de la familia son una importante base para entender las diferentes realidades de los niños en nuestro territorio. Esto se demuestra de forma particular en su investigación en torno a una problemática conocida como "el gaminismo”, en la que realizó una aproximación a la compleja realidad familiar que se gestaba en las crecientes urbes del país y daba como resultado la presencia de niños en las calles. Siguiendo esta línea de trabajo encontramos las investigaciones realizadas por Cecilia Muñoz y Ximena Pachón (1998, 1990, 2002, 2019) y por Pachón (2009b), enfocadas en el desarrollo de la infancia en Bogotá a lo largo del siglo XX. Estas investigadoras han abordado temas como la mortalidad infantil, el abandono, la vida en la calle y la vinculación de los niños a la guerra a partir de la revisión de fuentes históricas y etnográficas. Más recientemente, se suma a este interés la discusión que Max Hering (2018) realiza en su obra 1892: un año insignificante, en un capítulo dedicado a los niños de la calle, quienes, 
criminalizados sistemáticamente, se convertían en objeto de trabajos supuestamente concertados - pero que eran más bien forzados - en las haciendas cafeteras del occidente de Cundinamarca y el Tolima.

\section{¿La bioarqueología también los ha escondido? La infancia en la bioarqueología}

Hasta hace pocas décadas, el rol de los niños se encontraba en un lugar secundario de los estudios arqueológicos, posiblemente a causa de la dificultad de observar evidencia material producida por ellos y, por ende, incluirlos en la discusión arqueológica (Lillehammer 1989). Esta condición periférica llevó a que estuvieran supeditados en la investigación al mundo de los adultos, y los convirtió en seres socialmente "incompletos", al negar su capacidad de actuar y, en consecuencia, de producir registro arqueológico (Politis 1998; Sacchi 2010). A estos individuos y sus artefactos se los tomó como inexistentes (Fernández 2010), como pequeños adultos o como elementos perturbadores del registro arqueológico de los mayores, al reutilizar o “jugar” con objetos desechados (Politis 1998).

Sin embargo, con los desarrollos del posprocesualismo y de la arqueología de género se ha producido un corpus de investigaciones que cuestionan este tipo de ideas (Fernández 2010). Estas nuevas líneas de trabajo han reconocido a los niños del pasado como activos productores de registro material y como seres dinámicos en la construcción de su propio mundo (Halcrow y Tayles 2011; Perry 2006); mientras que otras han enfatizado el potencial de contribuir al entendimiento de la historia social, al permitirnos ver aspectos como la transmisión de la cultura entre generaciones (Lillehammer 1989).

Si se compara con otras disciplinas, la infancia como objeto de estudio apareció de forma tardía en la arqueología. Las primeras investigaciones se dieron en la década de 1970 en los países escandinavos, con los trabajos pioneros de Bonnichsen, Gräslund y Rolfsen (Herrero 2013). Sin embargo, fue Grete Lillehammer quien en 1989 visibilizó la importancia del mundo de los niños y su papel social, y planteó las primeras formas de estudio desde la arqueología. Para aquel momento, los niños aparecieron en investigaciones ligadas a temas de género y poco a poco aumentó el número de publicaciones sobre la infancia en las sociedades del pasado que los ubicaron como objeto central de la investigación, con una mayor elaboración analítica sobre aspectos relacionados con la cultura material y los contextos funerarios de la infancia (Lillehammer 2015). 
Estas primeras investigaciones permanecieron de forma aislada hasta entrada la primera década del siglo XXI, cuando la temática se volvió recurrente en congresos, seminarios y monografías (Rodríguez 2015). En el 2004 se inició una serie de congresos dedicados a la arqueología de la infancia en Europa y Norteamérica, y en el 2005 se creó la Society for the Study of Childhood in the Past (SSCIP), sociedad internacional y multidisciplinar que promueve los estudios de los individuos infantiles y que ha desarrollado publicaciones y encuentros académicos (Rodríguez 2015). En consecuencia, el número de publicaciones relacionadas con la infancia ha aumentado alrededor del mundo y ha ampliado, cada vez más, el panorama y las estrategias metodológicas para abordar inquietudes como las condiciones de vida de estos sujetos, su producción material y las formas de transmisión del conocimiento visibles en el registro arqueológico (Lillehammer 2015).

Investigaciones recientes han contribuido con herramientas metodológicas para analizar este tipo de casos (Agarwal y Beauchesne 2011; Mays et al. 2017; Perry 2006; Pinhasi y Mays 2008). Ha sido clave la publicación de trabajos como Developmental Juvenile Osteology, de Scheuer y Black (2000); Osteology of Infants and Children, de Baker de Dupras y Tocheri (2005), y The Bioarcheology of Children, de Lewis (2007), que han ampliado las posibilidades metodológicas, lo que también se evidencia en la revisión bibliográfica realizada por Mays et al. (2017) de los artículos publicados entre el 2006 y el 2015 en varias revistas de antropología física y osteoarqueología, en las cuales se manifiesta un creciente interés por la infancia, principalmente en países de Europa Occidental. Según esa revisión, uno de cada cinco artículos se enfocó de forma sustancial en los restos infantiles, y los principales temas de investigación fueron los estudios paleopatológicos y de isótopos estables.

En temas como el destete y la dieta infantil se han utilizado análisis químicos (isotópicos) de huesos y dientes, y análisis macroscópicos de patrones de desgaste dental, mientras los estudios paleopatológicos han encontrado en los estudios de ADN una herramienta particularmente fructífera para la investigación de enfermedades infecciosas que pueden ser difíciles de identificar en los esqueletos de los niños. No obstante, el análisis de Mays et al. (2017) también indicó que temas más tradicionales, como el estrés fisiológico infantil ${ }^{6}$, visto a través de indicadores como la criba orbitaria, la hiperostosis porótica, la hipoplasia

$6 \quad$ El estrés fisiológico se refiere a los indicadores de respuesta biológica, evidentes en los restos óseos y dentales, a presiones ambientales o sociales que sufren los individuos, lo que permite reconstruir parte de la relación de los niños en su contexto ambiental y sociocultural (Agarwal y Beauchesne 2011; Halcrow y Tayles 2011; Pinhasi y Mays 2008; Temple y Goodman 2014). 
del esmalte y el retraso en el crecimiento, siguen siendo un foco importante de estudio y debate gracias a la evidencia que brindan sobre las condiciones de vida de los niños.

Por otro lado, muchos estudios han ido más allá al reconocer el papel activo y la agencia social de los niños en la conformación de su entorno, al considerar la participación de estos en la producción cultural y material de sus comunidades (Halcrow y Tayles 2011; Perry 2007). Lo mismo ocurrió con algunas investigaciones que reconocen su intervención en actividades usualmente asociadas a los adultos, como la elaboración de artefactos (Politis 1998), la guerra o el trabajo (Lewis 2016; Perry 2006). Durante la última década ha habido un creciente interés por acercar la arqueología a la teoría social, lo que ha generado lo que Mays et al. (2017) denominan bioarqueología social, más teóricamente informada. Este enfoque se relaciona tanto con las interpretaciones teóricas y las construcciones sociales del cuerpo, como con el impacto que los procesos sociales y ambientales tienen sobre este y que son visibles en el registro bioarqueológico. Esto ha permitido explorar también los efectos de formas específicas de estrés inducido socialmente sobre los niños.

\section{¿Han estado escondidos bajo tierra? La arqueología latinoamericana y colombiana}

Para el caso latinoamericano se destacan trabajos como el estudio etnoarqueológico realizado por Politis (1998), a partir de comparaciones etnográficas entre los nukak maku de la Amazonía colombiana, con respecto a la participación infantil en la producción de objetos y el registro arqueológico de los sitios de la región pampeana de Argentina. El autor identificó posibles escenarios de participación de los niños en la producción de elementos visibles en el registro arqueológico ${ }^{7}$ y demostró el potencial de este objeto de estudio y la influencia de la infancia en el mundo material. Politis (1998) planteó, además, posibles salidas metodológicas

$7 \quad$ Politis (1998) identifica tres tipos de artefactos: 1) los confeccionados exclusivamente para jugar, que tienen un diseño específico; 2) los que copian la forma de aquellos de los adultos pero que tienen un tamaño menor y que son usados en funciones similares a las del adulto o con fines lúdicos; 3) los de los adultos, enteros o fragmentados, que son utilizados con fines lúdicos. 
a los problemas asociados al estudio de individuos infantiles. Para él una buena herramienta consiste en combinar varios tipos de fuentes, puntualmente de corte etnográfico, para poder entender el registro material que dejan estos individuos.

Siguiendo esta misma línea temática, encontramos investigaciones encaminadas a estudiar los juguetes y la producción material de los niños desde una perspectiva que vincula la enseñanza y transmisión cultural de comunidades prehispánicas. Entre estos, Fernández (2010) realizó un interesante análisis de las puntas de proyectil reportadas en la literatura arqueológica de los Andes centro-sur del periodo Arcaico (10.000-4000 a. P.) y del periodo Formativo (1000 a. C.400 d. C.), con el objetivo de visibilizar la actividad infantil y estudiarla como agente activo en la generación del registro arqueológico. A partir de la reevaluación de las características morfológicas de estos artefactos líticos, la autora logró identificar que algunos tipos de puntas de proyectil en realidad corresponden a artefactos utilizados por niños. Durante los últimos años, países como México se han convertido en un importante referente en la arqueología y bioarqueología de la infancia. Múltiples trabajos realizados tanto por investigadores con reconocida trayectoria como por estudiantes de maestría o doctorado (Hernández y Márquez 2010; Márquez 2006; Márquez y Sosa 2016; Pérez 2014) han generado un volumen creciente de estudios de caso, de propuestas metodológicas y de discusiones teóricas encaminados a entender diferentes aspectos de la infancia mesoamericana, es decir, las condiciones de vida, las tasas de mortalidad, la socialización, la transmisión de saberes, las migraciones, el sacrificio, entre los asuntos más destacados. Dentro de las discusiones resaltan aquellas orientadas a entender el papel social de los niños y a cuestionar el modo en que la arqueología ha asumido el tema de la infancia. Ejemplo de ello es la crítica que hacen Márquez y González (2006) a la perspectiva binaria y estructuralista de la arqueología tradicional, que ha definido a los niños como lo pasivo, ignorante y doméstico, en oposición a los adultos, asociados a la cultura y lo público. Para ellos y otros investigadores mexicanos, el problema no es que los niños estén ausentes o sean invisibles en el registro arqueológico, sino la falta de modelos teóricos adecuados para verlos.

En cuanto a la arqueología colombiana, encontramos muy pocos trabajos centrados en la infancia; sin embargo, tal como ocurre a nivel internacional, es posible rastrear su presencia en varias investigaciones arqueológicas. En la mayoría de los casos la infancia se presenta de modo tangencial, complementando o acompañando trabajos enfocados en la población en general, por lo que raramente se ha tomado como una variable de análisis relevante. No obstante, así sea de un modo parcial, estos trabajos han recogido valiosa información sobre el 
pasado de la infancia. A continuación, se presentan algunos estudios que abarcan diferentes periodos y nos ayudan a caracterizar el modo en que ha sido abordado el estudio arqueológico sobre la infancia.

Es importante aclarar que durante la revisión de estos trabajos arqueológicos notamos una tendencia hacia el análisis de los restos óseos infantiles, sin embargo, existen algunos estudios, como el de Vásquez (2014), que consiste en un análisis iconográfico de los materiales arqueológicos - principalmente cerámicos - de la cultura Tumaco-La Tolita, con el propósito de visibilizar la presencia de los niños en el registro material y comprender la concepción de infancia dentro de esta cultura. De hecho, identifica en las formas y en la decoración de los elementos cerámicos lo que serían representaciones de la infancia, la maternidad y el cuidado de los padres. Según esta autora, la iconografía de la maternidad y la infancia se identifica en las representaciones de mujeres en estado de embarazo o en labor de parto, así como en imágenes de mujeres en la tarea de amamantar a sus hijos y otras representaciones "familiares" (51).

En cuanto a estudios bioarqueológicos, en primer lugar encontramos los entierros infantiles identificados por Gonzalo Correal (1990) en el sitio arqueológico de Aguazuque, ubicado en el actual municipio de Soacha. En este se hallaron un conjunto de contextos funerarios y rituales, tanto individuales como colectivos, de individuos infantiles. Si bien Correal no ahonda en los resultados del análisis de estos individuos, la información recabada le permitió plantear la hipótesis de un aumento en la mortalidad infantil entre el 3400 y el 2800 a. P.

Juliana Gómez y Bibiana Cadena (2011) también involucraron individuos infantiles de este periodo en su estudio sobre el potencial informativo de la estimación de la estatura a partir de individuos recuperados en contextos precerámicos de cazadores-recolectores (provenientes de los sitios Checua y Tequendama) y horticultores tempranos (del sitio Aguazuque) que habitaron la sabana de Bogotá entre 8500 años a. P. y 3000 años a. P. aproximadamente. Después del análisis osteométrico de los huesos largos, como el húmero, la tibia y el fémur de 51 individuos adultos y 9 infantiles, concluyeron que la estatura, vista como un indicador de salud y nutrición, se mantuvo estable durante más de cinco milenios, a pesar de los cambios en los modos de subsistencia. Sin embargo, no proporcionan una reflexión detallada sobre los individuos infantiles que fueron incluidos en su muestra.

Por otro lado, el interés de José Vicente Rodríguez por las condiciones de vida de los pueblos prehispánicos lo llevó a realizar numerosos estudios en diferentes zonas de Colombia en los que se incluyeron individuos infantiles. En el Valle del Cauca hizo un análisis comparativo entre varias muestras arqueológicas 
que abarcan los periodos denominados Ilama, Yotoco y Sonso, que en conjunto abarcan desde el I milenio a. C. hasta la Conquista española. Este trabajo no estuvo particularmente enfocado en la población infantil, sino que intentó reconstruir el panorama general de estas comunidades, por lo cual los niños son vistos como uno de los grupos generacionales de estudio. No obstante, a partir del estudio de las patologías presentes en los restos óseos y del estudio de las características demográficas de esta población, el autor identifica un descenso en las tasas de mortalidad infantil, lo que podría indicar que los niños de las sociedades agroalfareras tempranas (II-I milenio a. C.) tenían una calidad de vida muy inferior a la de las tardías, inmediatamente anteriores al contacto español (Rodríguez 2005).

Siguiendo este mismo enfoque, en el sur del Alto Magdalena fueron analizados 486 registros funerarios correspondientes a los periodos Precerámico (entre los milenios X-II a. C.), Formativo (entre el I milenio a. C. y el siglo I d. C.), Clásico Regional (siglos II-IX d. C.) y Reciente (siglos X-XVI d. C.), con la intención de comprender mejor las dinámicas de cambio social a lo largo del tiempo y las condiciones de vida de la población (Rodríguez et al. 2017). Dentro de esta muestra se encontró una buena cantidad de individuos infantiles en los diferentes periodos, aunque la mayor parte estaba en regular o mal estado de preservación, lo que dificultó el análisis. Sin embargo, se identificó un trato especial hacia los individuos menores de cinco años, representado en un patrón funerario particular; se determinó que la edad del destete estaría cercana a los tres o cuatro años y se identificó el posible final de la infancia a temprana edad, al encontrar los restos de una niña de aproximadamente nueve años que murió en estado de embarazo. Esta niña, a pesar de su corta edad, fue enterrada como una mujer adulta en tumba de pozo y cámara (Rodríguez et al. 2017, 186).

En cuanto al Bajo Magdalena, Rojas-Sepúlveda y Martín (2015) reportan el estudio de la colección del Museo Mapuka de la Universidad del Norte, en la cual se encuentran los restos óseos excavados por Angulo Valdez entre las décadas de 1950 y 1980 en los sitios Malambo, Ciénaga de Guájaro, Tubará y Valle de Santiago. Si bien el tamaño de la colección (41 individuos y varios conjuntos de elementos óseos mezclados, cuyo número mínimo de individuos fue 45) no permite realizar aproximaciones poblacionales, se reporta en este trabajo la presencia de restos infantiles en varios contextos con algunas muestras de anomalías óseas de origen probablemente carencial (Rojas-Sepúlveda y Martín 2015). Con todo, los niños en este análisis también hacen parte de una pregunta general y no son tratados en específico. 
Otro ejemplo de este tipo de investigaciones lo encontramos en torno a la aldea muisca conocida como Tibanica, ubicada entre el 1200 y el $1600 \mathrm{~d}$. C. En este sitio arqueológico se recuperaron numerosas evidencias a partir de las cuales se pudieron abrir múltiples líneas de trabajo, que van desde las formas de organización social y jerarquía hasta la dieta y condiciones de vida de estos individuos (Langebaek et al. 2015). Si bien dentro del trabajo general con esta colección no se hace énfasis específico en los individuos infantiles, en el trabajo realizado por Lucero Aristizábal (2010) sí, pues su propósito fue caracterizar bioantropológicamente restos óseos y genéticos para encontrar posibles evidencias asociadas a la práctica de sacrificios humanos mediante un contraste con relatos de los cronistas sobre el sacrificio infantil. Buscó, además, identificar la procedencia de los individuos sacrificados. No obstante, no se encontraron evidencias de muerte violenta o algún signo claro de sacrificios humanos. Esta investigación es una de las pocas que se han realizado en el país enfocadas en la población infantil, y aunque resalta la importancia que podían tener los niños muiscas dentro de su sociedad al ser los sujetos escogidos para este tipo de sacrificios, el análisis se centra en la cuestión del sacrificio y no profundiza en las características y condiciones generales de vida de la infancia muisca.

José Vicente Rodríguez (2011), después de comparar los relatos de los cronistas y diferentes contextos funerarios infantiles muiscas (ubicados en Monquirá y Sogamoso, en el departamento de Boyacá, y Madrid, Soacha — sitio Tibanicaentre otros, sin especificar las muestras de cada uno de ellos), tampoco encuentra evidencias de sacrificio infantil entre esta población. Sin embargo, plantea que la presencia de niños en el interior de las viviendas podría tratarse de una ofrenda y no de un sacrificio ligado a una muerte violenta. El autor reconoce además un trato preferencial hacia los individuos infantes ya que, sin importar su rango, presentan características particulares en sus contextos funerarios; el hecho de que se encontraran bajo el piso de las casas podría ser interpretado como un ritual de consagración de la vivienda aprovechando la pureza infantil (Rodríguez 2011).

Sobre esta población también se encuentra el trabajo de Carrillo (2016), que analizó los resultados del estudio de 150 individuos infantiles y sus contextos funerarios provenientes del sitio arqueológico conocido como Nueva Esperanza, en el municipio de Soacha (Cundinamarca). A partir del análisis de estas variables, identifica el inicio de la participación de los niños en las labores de producción de herramientas líticas hacia los diez años y una diferencia de la orientación geográfica en los contextos funerarios de los individuos de entre once y quince años - especialmente en el periodo Muisca temprano-, los cuales, a diferencia de los de las demás edades, estaban orientados hacia el norte. 
Por otro lado, encontramos menos trabajos arqueológicos en los que se reflexione sobre los individuos infantiles que habitaron durante la Colonia. Sin embargo, investigaciones como las realizadas por Rivera $(2004,2014)$ han incluido el análisis de restos óseos infantiles de este periodo. En un primer momento, Rivera (2004) estudió 22 individuos infantiles provenientes del Claustro de Santo Domingo de la ciudad de Cartagena de Indias, cuyo análisis consistió en la determinación del sexo, la estimación de la edad y de posibles anomalías óseas. Por el estado de preservación de los restos, este último aspecto no pudo ser estudiado a profundidad. No obstante, dado que la mayor parte de estos individuos eran menores de cinco meses, esto le permitió concluir que entre los siglos XVI y XVIII existió en la ciudad una elevada mortalidad durante los primeros meses de vida.

Igualmente, en la investigación de Rivera (2014), realizada durante las labores de restauración de la iglesia Nuestra Señora de la Candelaria en Bogotá, se analizaron cerca de 117 individuos, lo cual corresponde al patrón funerario usual para este periodo. Dentro de esta muestra se identificaron 22 individuos infantiles y se encontró una importante proporción de neonatos (31,82 \%). Sin embargo, por el estado de preservación de los restos y por la falta de anomalías óseas visibles en estos individuos, la investigación centró su atención en los adultos.

Finalmente, para poblaciones históricas encontramos la investigación realizada por Rojas-Sepúlveda y Rodríguez (2017), cuyo objetivo fue documentar el perfil bioantropológico de un grupo de individuos inhumados en la parte occidental del Cementerio Central de Bogotá entre finales del siglo XIX y mediados del siglo XX, enfocándose en la distribución por sexo y por edad de los indicadores de estrés observados en los restos óseos. Como resultado de esta labor se pudieron reconstruir diversos aspectos de la vida de las personas inhumadas en este sector del cementerio, entre ellos, que las precarias condiciones de higiene y una dieta hipercalórica e hipoproteica produjo una considerable presencia de caries y cálculo dental, así como la presencia de indicadores de privación; la alta prevalencia de enfermedad articular degenerativa y de cambios entesiales indicaría una intensa actividad laboral y las duras condiciones de vida habrían producido una baja esperanza de vida al nacer; alrededor de 40 años (Rojas-Sepúlveda y Rodríguez 2017).

De los 7.103 individuos analizados en este estudio, 114 corresponden a individuos infantiles y cerca de 5.677 piezas óseas infantiles no pudieron ser individualizadas. Entre estos individuos, la muerte se presentó principalmente entre los cero y los cinco años (Rojas-Sepúlveda y Rodríguez 2017). A causa del gran tamaño de la colección estudiada, la investigación no profundizó más en aspectos particulares de esta población, por lo que posteriormente se desarrolló el análisis detallado y sistemático del material óseo infantil para comprender con 
mayor claridad sus condiciones de vida y su lugar en la sociedad (Coy 2019). En este estudio fueron analizados 113 individuos pertenecientes a un sector de la población que se consideraba en la documentación histórica como de escasos recursos, pues el globo B del Cementerio se denominó por largo tiempo “el cementerio de los pobres” (Rojas-Sepúlveda y Rodríguez 2017). De acuerdo con las fuentes históricas y literarias, estos individuos habrían sufrido altos niveles de mortalidad, asociada a la mala alimentación (Coy 2019). Como resultado del análisis conjunto de los restos óseos y de las fuentes históricas, se logró identificar una relación directa entre el contexto sociocultural y ambiental que vivieron estos individuos y las condiciones de vida y muerte asociadas. Por un lado, se determinaron algunos indicadores de procesos de estrés en el organismo, principalmente hiperostosis porótica, hipoplasias del esmalte, caries y desgaste dental, que estarían relacionados con una mala alimentación basada en el consumo de alimentos abrasivos, como por ejemplo algunas verduras de hoja verde, y a la posible persistencia de la costumbre de moler el maíz en piedras de moler, y poca higiene oral, aparte de repetidas epidemias. Además, la presencia de huellas de corte asociadas a procesos de necropsia y fracturas indicaría que algunos murieron de forma violenta o por causas desconocidas, que pudieron haber llamado la atención de los médicos forenses de la época: el presunto descuido de las madres o la sospecha de que el individuo hubiera muerto por razones "no naturales"; el escaso acceso a los centros de salud seguramente propiciaba la muerte de los individuos más jóvenes fuera de estos espacios y, por ende, la suspicacia sobre sus causas no permitía descartar el infanticidio (Muñoz y Pachón 2002). Al revisar las temáticas trabajadas en diferentes revistas de arqueología colombianas y latinoamericanas durante los últimos diez años ${ }^{8}$, notamos que menos del $1 \%$ de los artículos publicados tiene a la infancia como su tema central.

Aunque es evidente un avance al empezar a tratar estos asuntos, esto puede ser interpretado tan solo como el inicio de una nueva línea de investigación; así, por ejemplo, el estudio de su cultura material y de los papeles sociales que los niños desempeñaban en el pasado sigue estando a la espera de ser realizado. En la mayoría de los casos, los niños han constituido un punto de análisis anexo al estudio de los adultos o no han podido ser estudiados con la profundidad necesaria por no ser tomados como un eje central de la investigación.

Se revisaron las publicaciones entre el 2007 y el 2017 en las siguientes revistas: Revista Colombiana de Antropología (Colombia), Maguaré (Colombia), Antípoda (Colombia), Boletín de Antropología (Colombia), Revista Memorias (Colombia), Revista de Estudios Atacameños: Arqueología y Antropología Sur Andinas (Chile), Revista Arqueología (Argentina), Revista de Estudios de la Cultura Maya (México), Chungara. Revista de Antropología Chilena (Chile) y Revista de Arqueología Histórica (Argentina). 


\section{¿Por qué es tan difícil encontrar a los niños? Obstáculos para su estudio}

La pregunta sería entonces cómo podemos abordar la infancia del pasado para ponerla en diálogo con las discusiones arqueológicas contemporáneas. En primer lugar, es necesario reconocer los limitantes y sesgos que existen dentro de la disciplina y que dificultan el estudio de la infancia. Lillehammer (2015) aclara que la infancia y el mundo de los niños se siguen pasando por alto en los análisis científicos. Aunque cada vez se identifican más discusiones teóricas de la arqueología en las que se empieza a evaluar el papel de la infancia en el pasado y el modo en que ha sido abordada en la disciplina, en la práctica los materiales de la infancia suelen pasar desapercibidos, desde su proceso de excavación hasta en las colecciones de los museos centradas principalmente en los adultos ${ }^{9}$.

Entre los obstáculos para incluir a los niños en la discusión arqueológica se encuentra que sus restos son vistos como periféricos y complicados de identificar en el registro arqueológico (Sacchi 2010). Esto se refuerza por la poca representatividad que tienen en las diferentes fuentes históricas, lo que dificulta verlos con claridad en el pasado, pues en no pocas ocasiones los registros se componen por fragmentos o huellas débiles, ya sea por lo complejo de ver su producción material o por la forma en que fueron asumidos por los adultos en su momento (Lillehammer 1989; Pollock 1990). En la mayoría de los casos, los niños fueron excluidos - junto con otros actores sociales - de la historia o versión oficial del pasado, y en ocasiones esta poca representatividad en las fuentes históricas y arqueológicas ha llevado a que muchos investigadores olviden su presencia, convirtiéndolos en sujetos ajenos a la producción social e histórica.

Una segunda dificultad se encuentra en los sesgos de los arqueólogos que ven a los niños desde su propia condición de adultos y desde su tiempo. En ese sentido, los modelos planteados sobre la infancia reflejan también las actitudes actuales del investigador con respecto a estos temas, lo que constituye un sesgo del que hay que ser conscientes al trabajar esta temática (Lillehammer 1989). Un ejemplo de esta situación se encuentra en los imaginarios actuales sobre la infancia; si bien hoy existen múltiples formas de verla, es dominante la idea de que los niños tienen —o deberían tener - un mundo aparte del de los adultos, en donde

$9 \quad$ Al respecto, la profesora Lourdes Márquez Morfín, en una de sus conferencias en la Universidad Nacional de Colombia, en septiembre del 2017, comentaba que en un museo alguna vez le mostraron algunas bolsas con lo que serían "huesos de animales", una rápida revisión de su parte le permitió advertir que se trataba de restos óseos infantiles. 
sean prioridades la escolaridad, el cuidado y el juego. Esto hace que esté presente la percepción de que los niños se encuentran aislados del resto de la sociedad, que no participan en los medios de producción o que no desempeñan un rol activo en las dinámicas sociales. Bajo esta idea, el niño es visto como un individuo incompleto o un adulto en formación, y el adulto es considerado el patrón ideal de persona. Según Unicef (2013), este modelo adultocentrista genera relaciones de poder y dominación basadas en la idea de superioridad de los adultos sobre los más jóvenes. El adulto goza de privilegios por el solo hecho de serlo, mientras que a los niños y jóvenes, por ser considerados incompletos a nivel social, se les niega su capacidad de actuar y de ser miembros plenos de la sociedad (Sacchi 2010). Esto reproduce un sesgo en el momento de interpretar el registro arqueológico de una sociedad, pues si no reconocemos el rol activo de los niños en el presente, difícilmente lo veremos en los niños del pasado.

Por último, se encuentran las limitaciones inherentes a los materiales estudiados. Por un lado, no existen diferencias claras entre el material cultural dejado por adultos y niños en el registro arqueológico, es decir, no es posible saber con certeza si algún artefacto fue usado por uno u otro grupo porque en la mayoría de los casos los elementos son similares (Sacchi 2010). Por otro lado, al ser individuos en crecimiento y formación, sus restos óseos requieren un tratamiento especial. Sus muertes tempranas reducen la posibilidad de encontrar evidencias en el material óseo de las posibles enfermedades que padecían o de sus condiciones de vida, ya que estos pudieron haber muerto antes de que ciertas enfermedades generaran marcas a nivel óseo (Lewis 2007; Wood et al. 1992), sin contar con la baja preservación que los restos óseos infantiles suelen presentar (White 2011).

Para superar estos inconvenientes, es necesario reconocer los sesgos y los desafíos metodológicos y analíticos a los que la bioarqueología de la infancia se enfrenta. En este caso, ajustar la mirada y el análisis en busca de los niños en el registro arqueológico permite no solo identificar sino también comprender la forma como estos individuos han estado presentes y han actuado en los diferentes contextos. 


\section{El que busca encuentra: nuevas perspectivas para el análisis de la infancia}

Con el propósito de alentar el estudio de la infancia del pasado, aquí expondremos algunas posibles vías de aproximación a estos sujetos. Partimos de una idea bastante básica: los niños estuvieron presentes en el pasado y, por ende, tuvieron que haber dejado huella en el registro arqueológico. Es por ello que el desafío trasciende el hallazgo de los niños en el registro arqueológico e histórico, ya que, si se mira con cuidado, se encuentran numerosas evidencias de ellos, en algunas ocasiones incluso en proporciones más elevadas de las esperadas. El reto está entonces en cómo abordarlos metodológicamente y superar los inconvenientes mencionados.

Un primer paso consiste en buscar sus huellas en todo tipo de fuentes para reunir la mayor cantidad de pistas sobre su vida. Investigaciones previas, principalmente aquellas interesadas en la historia de la infancia, han usado diferentes tipos de registros para ver a los niños, entre ellos se destacan la iconografía, la literatura, los diarios y la prensa (Ariès 1987; DeMause 1982; Muñoz y Pachón 2002; Pollock 1990). Como afirma Pollock (1990), existe una variedad de fuentes en donde se puede encontrar a los niños, aunque ninguna de ellas brinda una historia completa de la infancia; entonces, se hace necesario unir todas las pruebas o fuentes para generar una imagen más completa e identificar los vacíos que ameritan ser investigados.

La arqueología, por su lado, ha combinado el estudio del registro arqueológico con fuentes etnográficas y etnohistóricas para interpretar la cultura material de los niños (Lillehammer 1989; Politis 1998). Lillehammer (1989) sostiene que, al existir tanta variedad en las vidas de los niños de diferentes culturas, para comprender el registro arqueológico de la infancia es fundamental una mirada etnocultural, que combine y complemente las evidencias materiales con otras fuentes, como suele hacerse con las analogías etnográficas.

Por su parte, la bioarqueología se centra en la idea de que no existe evidencia más directa de la biología de la persona que su propio cuerpo. En el caso de poblaciones del pasado, el esqueleto es el portador de las huellas del modo de vida del individuo y, en conjunto, de la población (Larsen 1999, 2002). El modelo aplicado, con algunas modificaciones (Temple y Goodman 2014), fue propuesto desde la paleoepidemiología por Goodman, Martin y Armelagos (1984) y plantea una sumatoria de factores en los que el ambiente puede ofrecer recursos limitados 
y otros agentes estresores ${ }^{10}$, la cultura puede inducir o atenuar los estresores y los factores que causan resistencia en el anfitrión ${ }^{11}$ (el individuo) pueden generar una interrupción de la homeostasis (equilibrio). El estrés como disrupción fisiológica puede resultar en indicadores, es decir, en signos (evidencias) de dicho estrés en huesos y dientes. Esto quiere decir que los signos se convierten en indicadores de la respuesta biológica a presiones ambientales o sociales. Existen indicadores de estrés no específico y otros de estrés específico. Los primeros son la mortalidad, el crecimiento de los individuos y los defectos en el esmalte dental que muestran momentos en que el crecimiento del diente se detuvo; y los segundos son los traumas óseos (fracturas y dislocaciones) y las reacciones periósticas -tales como las formaciones anormales en superficies óseas- (Goodman et al. 1984). Así, además de plantear que el estudio del material óseo es indispensable, la bioarqueología insiste en la importancia de investigar el contexto a través de la evidencia arqueológica y de otras líneas de evidencia disponibles, como los documentos en contextos históricos o los estudios paleoambientales (Buikstra y Beck 2006; Civera 2010; Larsen y Walker 2010).

Frente a algunas críticas que surgieron con respecto a la interpretación de la presencia o ausencia de los indicadores de estrés utilizados (Wood et al. 1992), se propuso que estudiarlos en conjunto, sin dejar de lado las circunstancias de existencia de la persona - lo cual implica el análisis de variables sociales, políticas, ecológicas y económicas-, podría corregir las falencias señaladas por los críticos (Mendonça de Souza, De Carvalho y Lessa 2003; Waldron 2009). De modo que para conocer las características de la población infantil del pasado, se debe partir del estudio de sus restos óseos y dentales (Mays et al. 2017) puesto que, al proporcionar evidencias de estrés en el individuo, se hace posible reconstruir parte de la relación de los niños con su contexto ambiental y sociocultural (Agarwal y Beauchesne 2011; Halcrow y Tayles 2011; Pinhasi y Mays 2008; Temple y Goodman 2014).

Es importante aclarar que únicamente es posible acercarnos a las enfermedades o condiciones que dejan marca en las estructuras óseas debido a que estas son mucho menos comunes que aquellas que solo afectan los tejidos blandos (Waldron 2009), máxime en individuos infantiles, cuya muerte pudo llegar

10 Los agentes estresores del ambiente pueden ser virus, bacterias o depredadores; desastres naturales, sequías, exceso de calor, exceso de frío, etc.

11 La resistencia puede ser biológica, particularmente genética (el individuo nace con resistencia a determinada enfermedad, por ejemplo), o puede estar ligada a la edad o al sexo. También se ha considerado de índole cultural, pues el acceso a los recursos y servicios de salud hacen la diferencia a la hora de resistir a un agente estresor. 
antes de que la enfermedad dejara evidencia en los huesos (Wood et al. 1992). No obstante, como se planteó antes, se vienen desarrollando y fortaleciendo diferentes metodologías de análisis en la bioarqueología, que van desde los estudios tradicionales sobre el estrés fisiológico infantil hasta los análisis especializados, con pruebas de ADN y estudios químicos de huesos y dientes. Estos nuevos enfoques han permitido comenzar a entender las múltiples relaciones que los niños entablan con su entorno físico y con el mundo adulto.

Además de contribuir al conocimiento que tenemos sobre los niños del pasado en el país, la aplicación de este enfoque favorecería el desarrollo de estándares bioantropológicos adecuados para nuestra población. Estudios anteriores, como el de Rojas-Sepúlveda y Rodríguez (2017), han resaltado los inconvenientes de utilizar metodologías y estándares elaborados para poblaciones norteamericanas o europeas, como ocurre a la hora de determinar la edad de los individuos a través de la longitud de los huesos largos. El desarrollo de técnicas y metodologías más acordes con nuestra población, además de ser útil en los estudios bioarqueológicos, facilitaría otro tipo de investigaciones, como aquellas relacionadas con las labores de identificación en la antropología forense del país ${ }^{12}$.

En términos generales, el tema de la infancia ha sido poco trabajado en la bioarqueología. En estudios previos, la edad fue tomada simplemente como una variable más y no como un principio importante de la organización social (Sacchi 2010). Sin embargo, la bioarqueología ha empezado a preguntarse por aquellas dinámicas sociales del pasado que dictan de qué modo son asumidos los niños por su grupo a lo largo de su corta vida y en el momento de su muerte (Lewis 2007; Sacchi 2010). El desafío está en aprovechar estas herramientas de análisis y visibilizar a estos sujetos que han permanecido escondidos, al reconocer su potencial a la hora de entender la complejidad de las dinámicas sociales del pasado, especialmente en aspectos como la transferencia de la cultura y la tradición de una generación a otra (Lillehammer 1989).

En consecuencia, en una perspectiva bioarqueológica de la infancia es necesario entender al individuo infante de un modo integral, sin reducirlo a un ente biológico desvinculado de las construcciones sociales generadas en torno a él (Márquez y González 2006). Para evitar esa situación es importante reconocer que categorías como la edad y el género, lejos de ser unívocas (e inequívocas), son construcciones sociales y por lo tanto ameritan ser investigadas en vez de asumidas

12 Solo por mencionar un caso, el estudio de la relación del tamaño de los huesos largos en individuos fetales a través de los ultrasonidos realizados a las madres gestantes colombianas permitiría conocer mejor las características de nuestra población y desarrollar estándares más adecuados para la determinación de la edad de estos individuos. 
anticipadamente. En especial, porque estamos acostumbrados a poner énfasis en la edad cronológica para clasificar a las personas, como ocurre con la distribución de los niños en los diferentes grados escolares, mientras que otras sociedades acostumbran a hacer estas clasificaciones según estadios de maduración, capacidades, habilidades, personalidad y otros atributos (Márquez y González 2006).

A la arqueología colombiana aún le resta un largo camino por recorrer para que los niños no pasen desapercibidos en los contextos arqueológicos. Es necesario reconocer su presencia y participación en la historia para comprender mejor la complejidad y variedad de dinámicas que los involucraron en las sociedades de su tiempo. Este artículo hace un llamado a realizar más investigaciones arqueológicas centradas en los niños para que dejen de estar escondidos en el registro material del pasado.

\section{Referencias}

Agarwal, Sabrina C. y Bonnie A. Glencross, eds. 2011. Social Bioarchaeology. Nueva York: Wiley-Blackwell.

Agarwal, Sabrina y Patrick Beauchesne. 2011. "It is not Carved in Bone”. En Agarwal y Glencross 2011, 312-332.

Aguirre, María. 2001. "Enseñar con textos e imágenes. Una de las aportaciones de Juan Amós”. Revista Electrónica de Investigación Educativa 3 (1): 1-19. http://www.redalyc.org/ articulo.oa?id=15503101

Ariès, Philippe. 1987. El niño y la vida familiar en el Antiguo Régimen. Madrid: Taurus.

Aristizábal, Lucero. 2010. "Informe final. Entierro de niños en una aldea muisca tardía: caracterización bioantropológica y genética de los individuos subadultos de una muestra proveniente del complejo funerario muisca de Tibanica (Soacha)". Inédito.

Baker, Brenda, Tosha Dupras y Matthew Tocheri. 2005. The Osteology of Infants and Children. College Station: Texas A\&M University Press.

Buikstra, Jane E. y Lane A. Beck. 2006. Bioarchaeology: The Contextual Analysis of Human Remains. San Diego: Elsevier.

Carrillo, Liliana. 2016. "Arqueología de la infancia: aproximaciones desde las prácticas funerarias en el sitio arqueológico Nueva Esperanza". En "Informe final 'Proyecto rescate arqueológico Subestación Nueva Esperanza”, t. III. Inédito.

Civera, Magalí. 2010. "Avances y retos en la determinación de la salud de poblaciones antiguas a partir de restos esqueléticos”. Estudios de Antropología Biológica 12 (2): 859-870. http://www.revistas.unam.mx/index.php/eab/search/search 
Correal, Gonzalo. 1990. Aguazuque: evidencias de cazadores, recolectores y plantadores en la altiplanicie de la cordillera Oriental. Bogotá: Fundación de Investigaciones Arqueológicas Nacionales, Banco de la República.

Coy Carrera, Laura. 2019. "Condiciones de vida de los individuos infantiles de finales del siglo XIX y mediados del siglo XX, a partir de los restos óseos del globo B del Cementerio Central-Bogotá". Tesis de Maestría en Antropología, Facultad de Ciencias Humanas, Universidad Nacional de Colombia, Bogotá.

DeMause, Lloyd. 1982. "La evolución de la infancia”. En Historia de la infancia, editado por Lloyd DeMause, 15-92. Madrid: Alianza.

Dunn, Patrick. 1982. "Ese enemigo es el niño: la infancia en la Rusia imperial”. En Historia de la infancia, editado por Lloyd DeMause, 419-443. Madrid: Alianza.

Fernández, María. 2010. "El flautista de Hamelin en los Andes centro-sur: aproximaciones a la arqueología de la infancia a través del estudio de puntas de proyectil”. Arqueología y Arte Rupestre 1: 29-44.

Gómez, Juliana y Bibiana Cadena. 2011. "La estatura como indicador de calidad de vida a través del tiempo. ¿Es posible comparar sin estándares?”. Virajes 13: 337-352. http://virajes.ucaldas.edu.co/downloads/Virajes13_14.pdf

Goodman, Alan, Debra Martin y George Armelagos. 1984. "Indicators of Stress from Bone and Teeth”. En Paleopathology at the Origins of Agriculture, editado por Mark Cohen y George Armelagos, 14-38. Orlando: Academic Press.

Grimal, Nicolás. 1996. Historia del antiguo Egipto. Madrid: Akal.

Gutiérrez de Pineda, Virginia. 1978. El gamín, su albergue social y su familia. Bogotá: Unicef.

Halcrow, Sian y Nancy Tayles. 2011. "The Bioarchaeological Investigation of Children and Childhood”. En Agarwal y Glencross 2011, 333-370.

Hardman, Charlotte. 2001. “Can There Be an Anthropology of Children?”. Childhood 8 (4): 501-517. https://doi.org/10.1177/0907568201008004006

Harris, Marvin. 1979. El desarrollo de la teoría antropológica. Historia de las teorías de la cultura. Madrid: Siglo Veintiuno.

Hering, Max. 2018. 1892: un año insignificante. Bogotá: Crítica; Universidad Nacional de Colombia.

Hernández, Patricia y Lourdes Márquez. 2010. "Los niños y las niñas del antiguo Xochimilco: un estudio de mortalidad diferencial”. Revista Española de Antropología Física 31: 39-52.

Herrero, Ana. 2013. "Sobre la infancia en la prehistoria: un análisis de las sociedades del interior peninsular entre el VI y III Milenio AC”. El Futuro del Pasado 4: 69-86. http://dx.doi. org/10.14516/fdp

Hirschfeld, Lawrence. 2002. “Why don’t Anthropologists like Children?”. American Anthropologist 104 (2): 611-627. https://doi.org/10.1016/j.socscimed.2013.12.020 
Justel Vicente, Daniel. 2012. "El estudio de la infancia en el mundo antiguo". En Niños en la Antigüedad. Estudios sobre la infancia en el Mediterráneo antiguo, editado por Daniel Justel Vicente, 15-29. Zaragoza: Universidad de Zaragoza.

Lancy, David. 2012. "Why Anthropology of Childhood? A Brief History of an Emerging Discipline”. AntropoChildren 1: 1-17. https://popups.uliege.be/2034-8517/index. php?id=932\&file $=1 \&$ pid $=918$

Langebaek, Carl, Alejandra Jaramillo, Lucero Aristizábal, Marcela Bernal, María Corcione, Luisa Mendoza, Luz Pérez, Freddy Rodríguez y Catalina Zorro. 2015. "Vivir y morir en Tibanica. Reflexiones sobre el poder y el espacio". Revista Colombiana de Antropología 51: 173-207. http://www.scielo.org.co/pdf/rcan/v51n2/v51n2a08.pdf

Larsen, Clark S. 1999. Bioarchaeology: Interpreting Behavior from the Human Skeleton. Cambridge: Cambridge University Press.

-. 2002. "Bioarchaeology: The Lives and Lifestyles of Past People". Journal of Archaeological Research 10 (2): 119-66. https://www.jstor.org/stable/41053183?seq=1\#page_scan_tab_contents

Larsen, Clark S. y Phillip Walker. 2010. "Bioarchaeology: Health, Lifestyle, and Society in Recent Human Evolution”. En A Companion to Biological Anthropology, editado por Clark S. Larsen, 379-394. Chichester; Nueva York: Blackwell Publishing. DOI: 10.1002/9781444320039.ch21

Lewis, Mary. 2007. The Bioarchaeology of Children. Nueva York: Cambridge University Press.

-. 2016. "Work and the Adolescent in Medieval England (AD 900-1550): The Osteological Evidence”. Medieval Archaeology 60 (1): 138-171. https://doi.org/10.1080/00766097.2016.1147787

Lillehammer, Grete. 1989. "A Child is Born. The Child's World in an Archaeological Perspective”. Norwegian Archaeological Review 22 (2): 89-105. https://doi.org/10.1080/00293652.19 89.9965496

-. 2015. " 25 Years with the 'Child' and the Archaeology of Childhood”. Childhood in the Past 8 (2): 78-86. https://doi.org/10.1179/1758571615Z.00000000030

Little, Michael y Robert Sussman. 2010. "History of Biological Anthropology”. En A Companion to Biological Anthropology, editado por Clark S. Larsen, 13-38. Nueva York: Blackwell Publishing.

Márquez, Lourdes. 2006. "Sacrificio de niños en Chichen Itzá o práctica funeraria”. En Los niños como actores sociales ignorados. Levantando el velo, una mirada al pasado, editado por Lourdes Márquez Morfín, 253-282. Ciudad de México: ENAN-INAH; Conaculta.

Márquez, Lourdes y Ernesto González. 2006. "La socialización de los niños en el pasado. Algunas reflexiones y propuestas en torno al tema”. En Márquez 2006, 51-73.

Márquez, Lourdes y María Viridiana Sosa. 2016. "Mortalidad de niños y sífilis congénita en la Ciudad de México en 1915”. Estudios Demográficos y Urbanos 31 (1): 177-206. http://www. scielo.org.mx/scielo.php?script=sci_arttext\&pid=S0186-72102016000100177

Mays, Simon, Rebecca Gowland, Sian Halcrow y Eileen Murphy. 2017. "Child Bioarchaeology: Perspectives on the Past 10 Years”. Childhood in the Past 10 (1): 38-56. https://doi.org/ 10.1080/17585716.2017.1301066 
Mendonça de Souza, Sheila, Diana de Carvalho y Andrea Lessa. 2003. "Paleoepidemiology: Is There a Case to Answer?”. Memorias do Instituto Oswaldo Cruz 98 (1): 21-27. https:// doi.org/10.1590/S0074-02762003000900005

Montgomery, Heather. 2009. "Children within Anthropology: Lessons from the Past”. Childhood in the Past 2 (1): 3-14. https://doi.org/10.1179/cip.2009.2.1.3

Muñoz, Cecilia y Ximena Pachón. 1990. "Los chinos bogotanos a comienzos de siglo (19001930)”. Revista Credencial Historia 12. http://www.banrepcultural.org/biblioteca-virtual/ credencial-historia/numero-12/los-chinos-bogotanos-comienzos-de-siglo-1900-1930

-. 1998. Historia de la niñez en Bogotá, 1990-1988. Informe final. Bogotá: Banco de la República; Fundación para la Promoción de la Investigación y la Tecnología.

—. 2002. Réquiem por los niños muertos: Bogotá siglo XX. Bogotá: Cerec; Hogares Club Michín.

—. 2019. Los niños de la miseria. Bogotá, siglo XX. Bogotá: Universidad Nacional de Colombia.

Pachón, Ximena. 2005. "Virginia Gutiérrez de Pineda y su aporte al estudio histórico de la familia en Colombia”. Maguaré 19: 247-272. https://revistas.unal.edu.co/index.php/maguare/article/view/10772

—. 2009a. “Dónde están los niños? Rastreando la mirada antropológica sobre la infancia”. Maguaré 23: 434-469. https://doi.org/10.2307/25597211

-.2009b. "La infancia perdida en Colombia: los menores en la guerra". Working Paper Series 15 (15): 1-21. http://pdba.georgetown.edu/CLAS\%20RESEARCH/Working\%20Papers/WP15.pdf

Pavez, Iskra. 2012. "Sociología de la infancia: las niñas y los niños como actores sociales”. Revista de Sociología 27 (27): 81-102. https://doi.org/10.5354/0719-529X.2012.27479

Pérez, Hugo. 2014. "Hacia una arqueología de la infancia: ¿a dónde fueron los niños en Teotihuacán?”. Tesis de maestría, Escuela Nacional de Antropología e Historia, Ciudad de México.

Perry, Megan. 2006. "Redefining Childhood through Bioarchaeology: Toward an Archaeological and Biological Understanding of Children in Antiquity”. Archeological Papers of the American Anthropological Association 15 (1): 89-111. https://doi.org/10.1525/ap3a.2005.15.89

-. 2007. "Is Bioarchaeology a Handmaiden to History? Developing a Historical Bioarchaeology”. Journal of Anthropological Archaeology 26 (3): 486-515. https://doi.org/10.1016/j. jaa.2007.02.002

Pinhasi, Ron y Simon Mays. 2008. Advances in Human Palaeopathology. Chichester: John Wiley \& Sons.

Politis, Gustavo. 1998. "Arqueología de la infancia: una perspectiva etnoarqueológica”. Trabajos de Prehistoria 55 (2): 5-19. https://doi.org/10.3989/tp.1998.v55.i2.300

Pollock, Linda. 1990. Los niños olvidados: relaciones entre padres e hijos de 1500 a 1900. Ciudad de México: Fondo de Cultura Económica.

Ríos, Clara. 1998. "El último emperador de China: una vida entre dos interpretaciones del mundo y entre dos conceptos de formación”. Revista Educación y Pedagogía 22: 29-48. http://aprendeenlinea.udea.edu.co/revistas/index.php/revistaeyp/article/view/5773 
Rivera, Javier. 2004. "Costumbres funerarias en la Cartagena colonial, siglos XVI-XVIII. Estudio en el Claustro de Santo Domingo”. Tesis de Antropología, Facultad de Ciencias Humanas, Universidad Nacional de Colombia, Bogotá.

-. 2014. "Espacios mortuorios y bioarqueología histórica en la iglesia La Candelaria en Bogotá”. Maguaré 28 (2): 147-174. https://revistas.unal.edu.co/index.php/maguare/article/ view/54729

Rodríguez Cuenca, José V. 1996. "Panorama de la antropología biológica en Colombia y su relación con el ámbito latinoamericano y mundial”. Maguaré 11-12: 75-102. https://revistas. unal.edu.co/index.php/maguare/article/view/14274

-. 2005. Pueblos, rituales y condiciones de vida prehispánicas en el Valle del Cauca. Bogotá: Universidad Nacional de Colombia.

—. 2011. Los chibchas: hijos del sol, la luna y los Andes. Orígenes de su diversidad. Bogotá: Universidad Nacional de Colombia; IDU.

Rodríguez, Celtia. 2015. "Arqueología de la infancia: niños y niñas en la prehistoria reciente de la región de Murcia a través de los restos funerarios”. Arqueología y Territorio 12 (enero): 49-62. https://dialnet.unirioja.es/servlet/articulo?codigo=5377078

Rodríguez-Flórez, Carlos y Ernesto Rodríguez-Flórez. 2010. "Reflexiones sobre las evidencias óseas y dentales halladas en contextos arqueológicos de Colombia en los últimos 65 años (1945-2010)”. International Journal of South American Archaeology 7: 40-53. http://ijsa. syllabapress.com/issues/articles/ijsa00041/ijsa00041.pdf

Rodríguez, Pablo y María Mannarelli. 2007. Historia de la infancia en América Latina. Bogotá: Universidad Externado de Colombia.

Rojas-Sepúlveda, Claudia. 2014. "Breve historia, balance y perspectivas de la bioarqueología en Colombia”. En Avances recientes en la bioarqueología latinoamericana, editado por Leandro Luna, Claudia Aranda y Jorge Suby, 3-33. Buenos Aires: Grupo de Investigación en Bioarqueología.

Rojas-Sepúlveda, Claudia y Juan Martín. 2015. "Bioarqueología del Bajo Magdalena, norte de Colombia, América del Sur”. Revista Colombiana de Antropología 51 (2): 89-120. https:// doi.org/10.22380/2539472X15

Rojas-Sepúlveda, Claudia y José V. Rodríguez. 2017. “Informe final: análisis bioarqueológico de contextos funerarios del Cementerio Central, Bogotá: vida y muerte a final del siglo XIX y principios del XX”. Inédito.

Sacchi, Mariana. 2010. “Algunos apuntes sobre la arqueología de la infancia: exploración de vías metodológicas para su definición”. Revista de Antropología Experimental 10: 281-292. https://revistaselectronicas.ujaen.es/index.php/rae/article/view/1953

Scheuer, Louise y Sue Black. 2000. Developmental Juvenile Osteology, vol. 5. San Diego: Elsevier Academic Press. https://doi.org/10.1109/MSP.2007.36

Sevilla, Alberto. 2012. "Morir Ante suum diem. La infancia en Roma a través de la muerte”. En Justel 2012, 199-232. 
Temple, Daniel y Alan Goodman. 2014. "Bioarcheology Has a 'Health’ Problem: Conceptualizing 'Stress' and 'Health' in Bioarcheological Research”. American Journal of Physical Anthropology 155 (2): 186-191. https://doi.org/10.1002/ajpa.22602

Unicef. 2013. Superando el adultocentrismo. Santiago de Chile: Unicef.

Vásquez, Camila. 2014. "Arqueología de la infancia: hacia una concepción emic de la infancia en la cultura Tumaco-La Tolita”. Trabajo de grado en Antropología, Facultad de Ciencias Humanas, Universidad Nacional de Colombia, Bogotá.

Waldron, Tony. 2009. Paleopathology. Nueva York: Cambridge University Press.

White, Tim. 2011. Human Osteology. San Diego: Academic Press.

Wood, James, George Milner, Henry Harpending y Kenneth Weiss. 1992. "The Osteological Paradox: Problems of Inferring Prehistoric Health from Skeletal Samples”. Current Anthropology 33 (4): 343-370. https://www.jstor.org/stable/2743861 